\title{
RECENT WESTERBORK OBSERVATIONS \\ OF HEAD-TAIL GALAXIES
}

\author{
G. K. MILEY, H. VAN DER LAAN, and K. J. WELLINGTON \\ Sterrewacht, Leiden, The Netherlands
}

\begin{abstract}
Some recent observations of head-tail galaxies are presented. The properties of these objects are reviewed and are shown to be consistent with the radio trail hypothesis.
\end{abstract}

\section{Introduction}

During the last few years, observations with the Cambridge One-Mile Telescope have defined a new class of relatively weak radio galaxies characterized by a peculiar elongated morphology (Ryle and Windram, 1968; Hill and Longair, 1971). The radio sources have a high brightness 'head' close to the optical galaxy and a narrow low brightness 'tail' which sometimes extends for many minutes of arc. All the head-tail galaxies at present known (Table I) appear to be associated with clusters of galaxies.

TABLE I

Head-tail radio galaxies

\begin{tabular}{lll}
\hline Object & Cluster & Identification \\
\hline NGC 1265 (3C 83.1) & Perseus & Ryle and Windram (1968) \\
IC 310 & Perseus & Ryle and Windram (1968) \\
WBK 0314+41 & Perseus & Miley et al. (1972) \\
NGC 4869 (5C 4.81) & Coma & Willson (1971) \\
3C 129 & Obscured & Hill and Longair (1971) \\
3C 129.1 & Hill and Longair (1971), Miley (1973) \\
NGC 7385 (PKS 2247+11) & Zw 2247+11 & $\begin{array}{l}\text { Schilizzi (private communication) } \\
\text { Ekers (private communication) }\end{array}$ \\
\hline
\end{tabular}

Here I shall first describe Westerbork observations of two of the most beautiful examples 3C 129 and NGC 1265. After comparing some characteristics which these objects have in common with 'normal' double radio galaxies, I shall conclude with some deductions about their nature and about the properties of the intergalactic medium. We shall see that all the data are consistent with the hypothesis that the galaxies are active radio sources and that the tails are merely trails left behind as these galaxies plough through a dense intra-cluster medium (Miley et al., 1972).

\section{2. $3 C 129$}

Figure 1 shows a $1.4 \mathrm{GHz}$ radio photograph of $3 \mathrm{C} 129$. The radio tail extends for nearly half a megaparsec from the parent galaxy. Note the double nature of both the 


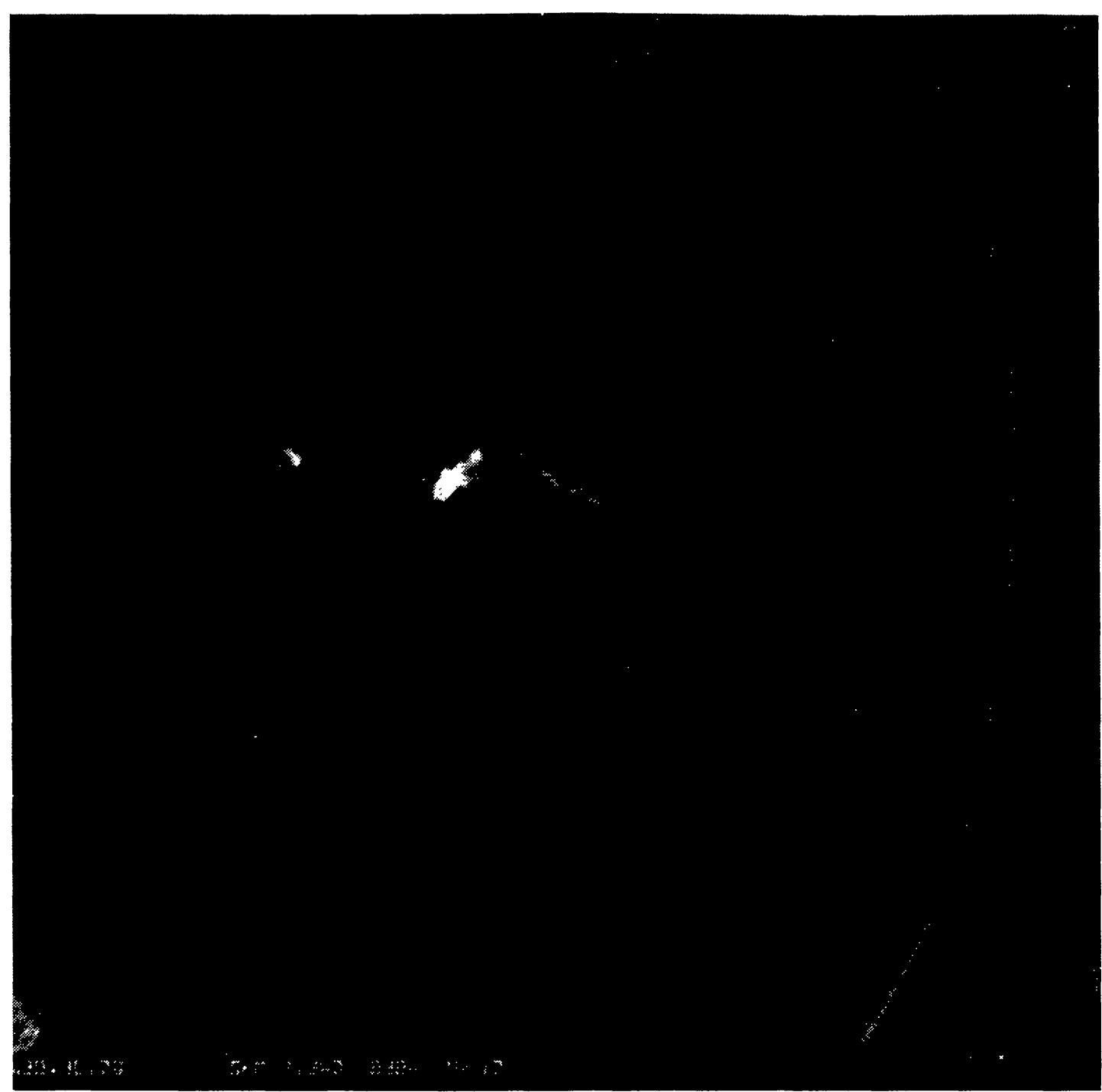

Fig. 1. 1.4 GHz radio photograph of 3C 129 (right) and 3C 129.1 (left) (from Miley et al., 1972). Bars indicate the position of the optical galaxy.

head and the tail. The polarization data are given in Figure 2 (Miley, 1973). The percentage polarization increases, and the radio spectrum gradually steepens, with distance along the tail (Figure 3). Compared with the head, the more relaxed tail has a higher polarization and steeper spectrum. It is interesting that for 'normal' double radio galaxies the more relaxed ones also appear to have relatively high polarizations and steep spectra (Miley and van der Laan, 1973).

\section{NGC 1265}

Figure 4 shows a $1.4 \mathrm{GHz}$ radio photograph of the galaxy NGC 1265 in the Perseus Cluster and Figures 5 and 6 give the $1.4 \mathrm{GHz}$ and $5.0 \mathrm{GHz}$ maps superimposed on the 


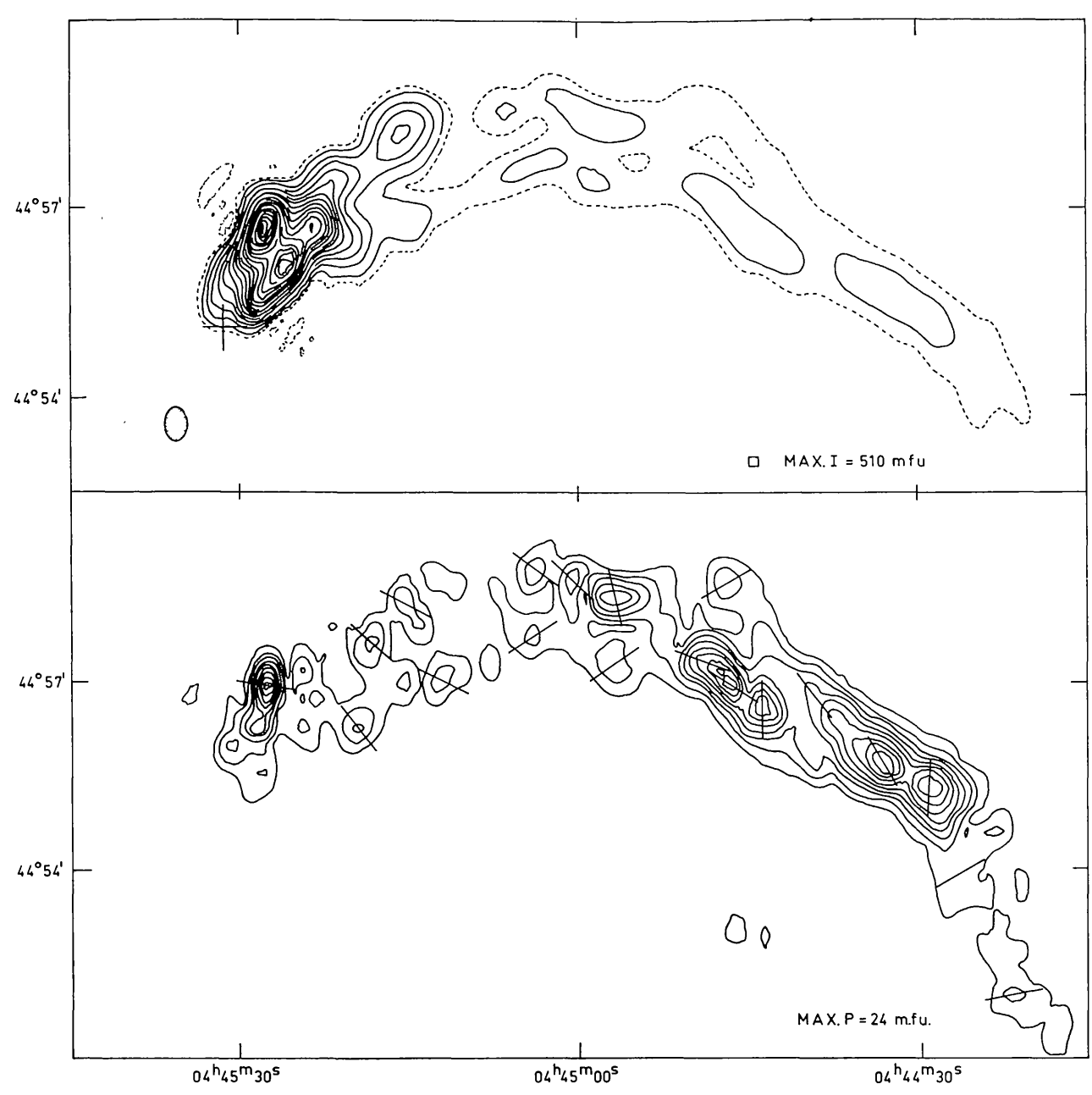

Fig. 2. The $1.4 \mathrm{GHz}$ total intensity (top) and polarization (bottom) distributions of $3 \mathrm{C} 129$ (from Miley, 1973).

Palomar prints. Here we also see the double nature of both head and tail. There is a compact opaque component near the centre of the optical galaxy. Note also the shape and discreteness of the head. Such a configuration is exactly what would be expected for a galaxy ejecting radio emitting blobs with a speed comparable to that of the galaxy (radial velocity $\approx 2300 \mathrm{~km} \mathrm{~s}^{-1}$ ) every few million years (Wellington et al., 1973).

\section{Comparison with 'Normal' Radio Galaxies}

The head-tail galaxies so far detected are intrinsically several hundred times weaker than the strongest 'normal' doubles. However, as we have seen, there are several characteristics common to both classes (Table II).

Because of these similarities it appears reasonable that head-tail galaxies should be explained within the context of theories of normal radio galaxies. The morphologies of 'normal' sources (Figure 7 of Miley and van der Laan, 1973) have been ex- 


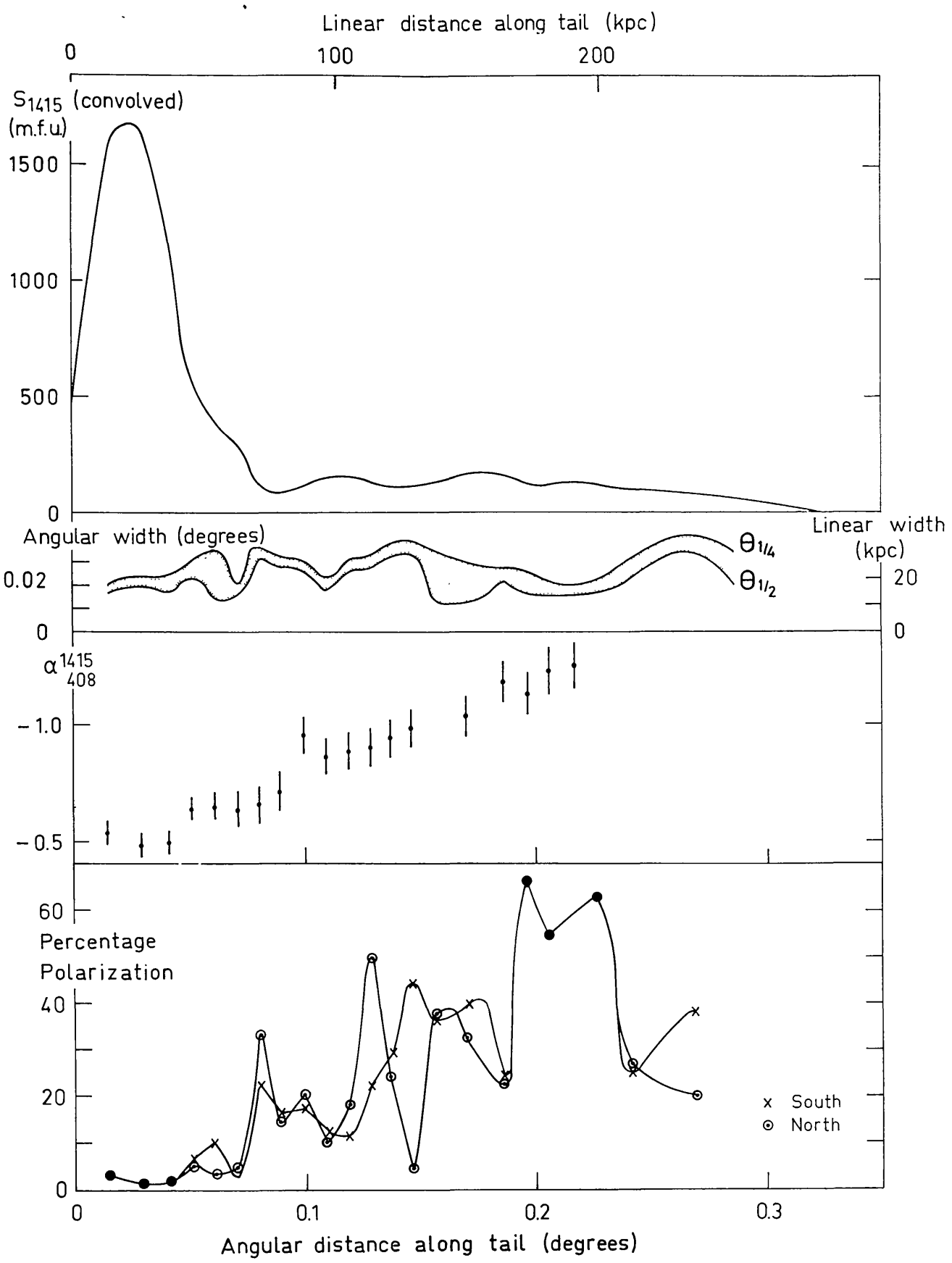

Fig. 3. Variation of brightness with spectral index and percentage polarization derived from $1.4 \mathrm{GHz}$ measurements on 3C 129 (See Miley, 1973).

plained in terms of ram pressure confinement by an extragalactic medium (3C 61.1, 3C 274.1, 3C 284, 3C 390.3, 3C 184.1) and subsequent adiabatic expansion (3C 310, 3C 314.1). Jaffe and Perola (1973) have discussed such models for radio sources ejected by moving galaxies. Their detailed radio trail models have been applied quite successfully to 3C 129 and NGC 1265. 


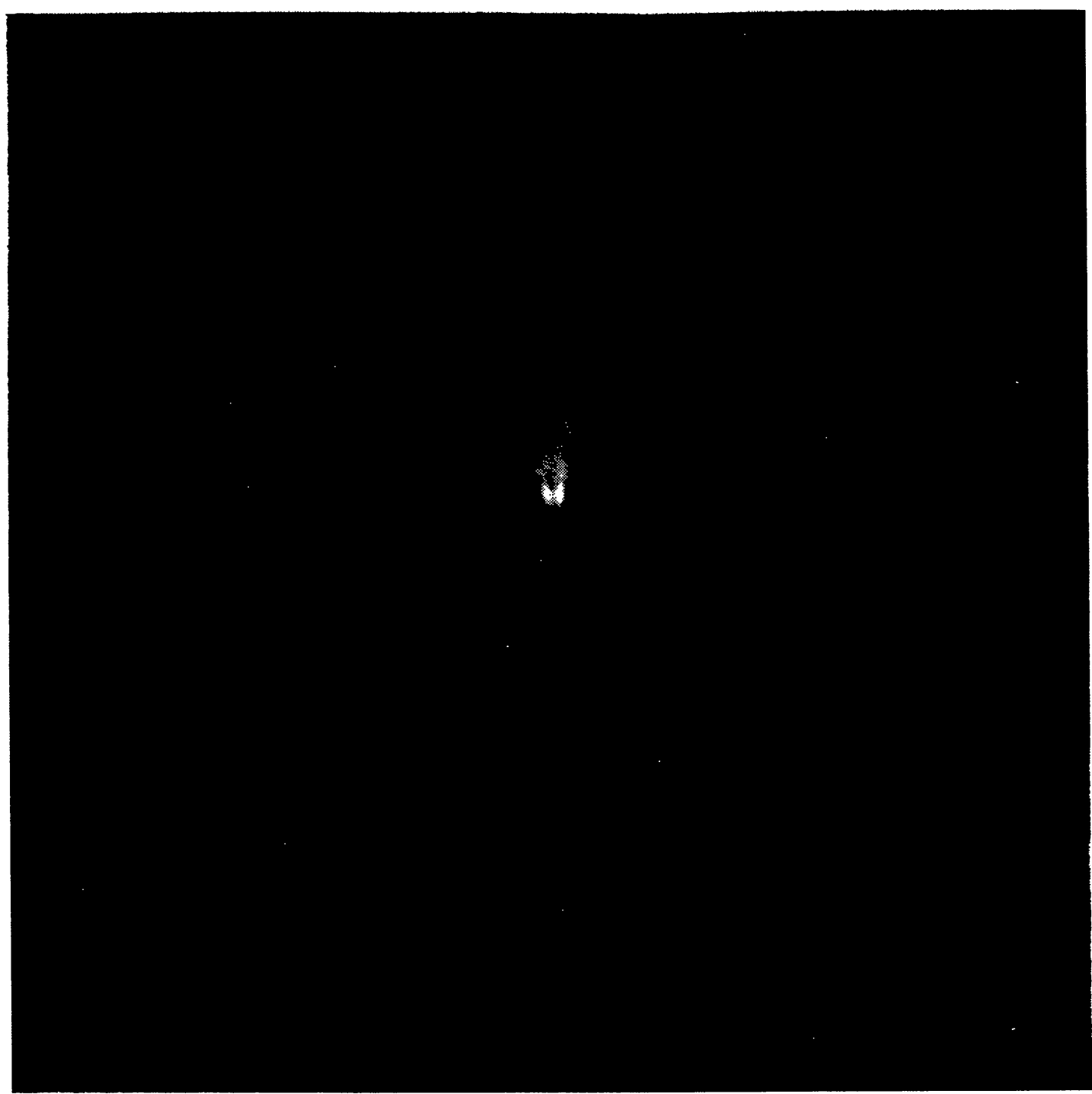

Fig. 4. $1.4 \mathrm{GHz}$ radio photograph of NGC 1265 (from Miley et al., 1972).

TABLE II

Similarities between 'normal' and 'head-tail' radio galaxies

\begin{tabular}{lll}
\hline Property & Normal & Head-tail \\
\hline $\begin{array}{l}\text { Morphology } \\
\text { Polarization }\end{array}$ & $\begin{array}{l}\text { Double } \\
\text { Larger in more relaxed sources }\end{array}$ & $\begin{array}{l}\text { Double head + double tail } \\
\text { Larger in more relaxed } \\
\text { regions of source }\end{array}$ \\
Spectrum & $\begin{array}{c}\text { Steeper for more relaxed } \\
\text { sources }\end{array}$ & $\begin{array}{c}\text { Steeper for more relaxed } \\
\text { regions of source }\end{array}$ \\
$\begin{array}{l}\text { Compact nuclear } \\
\text { sources with } \\
\text { flat spectra }\end{array}$ & Often present & Present in 3C 129, \\
& & NGC 1265 and IC 310 \\
\hline
\end{tabular}




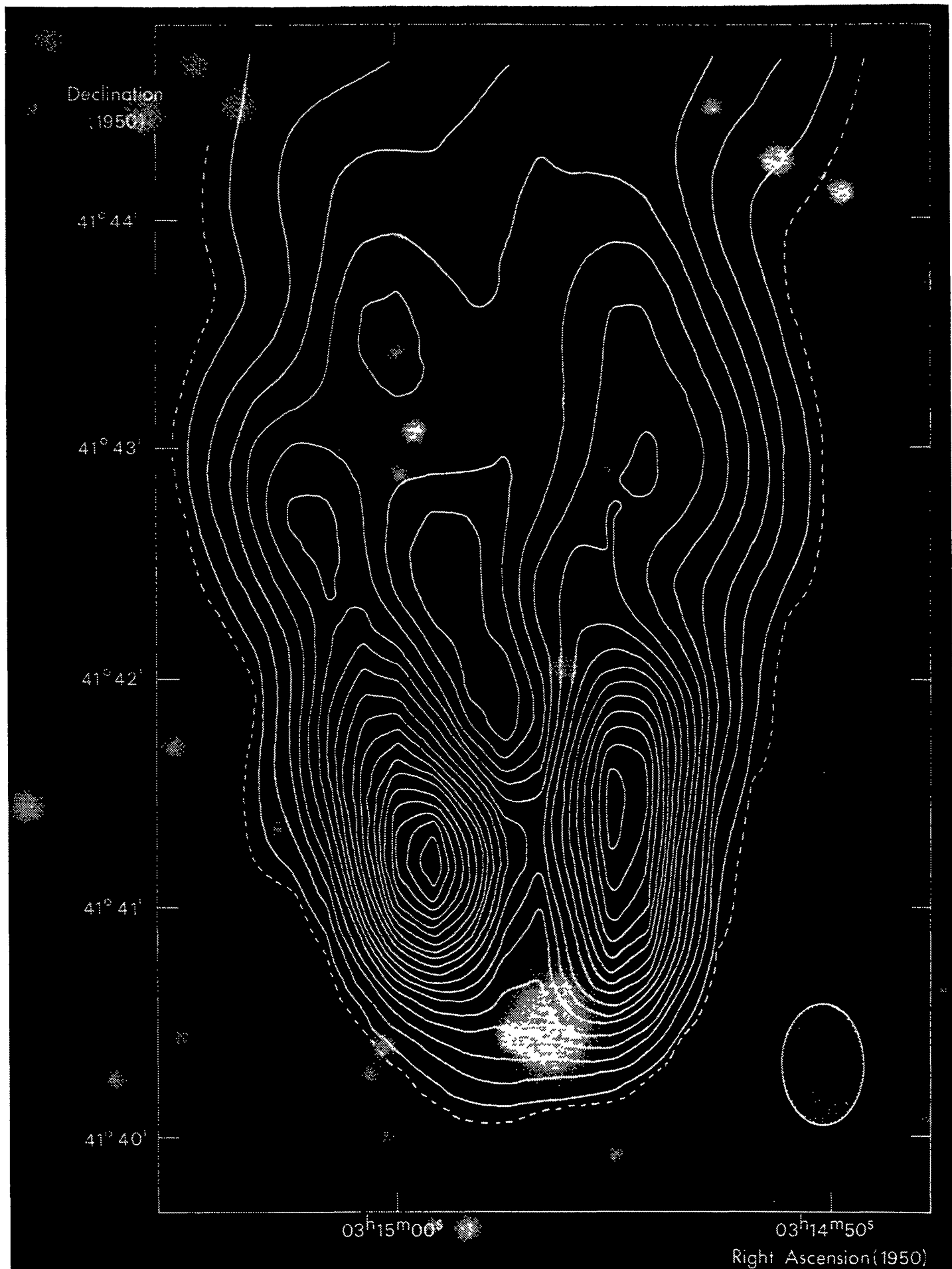

Fig. 5. $1.4 \mathrm{GHz}$ contour map of the head of NGC 1265 superimposed on the blue Palomar Sky Survey print (from Wellington et al., 1973). 


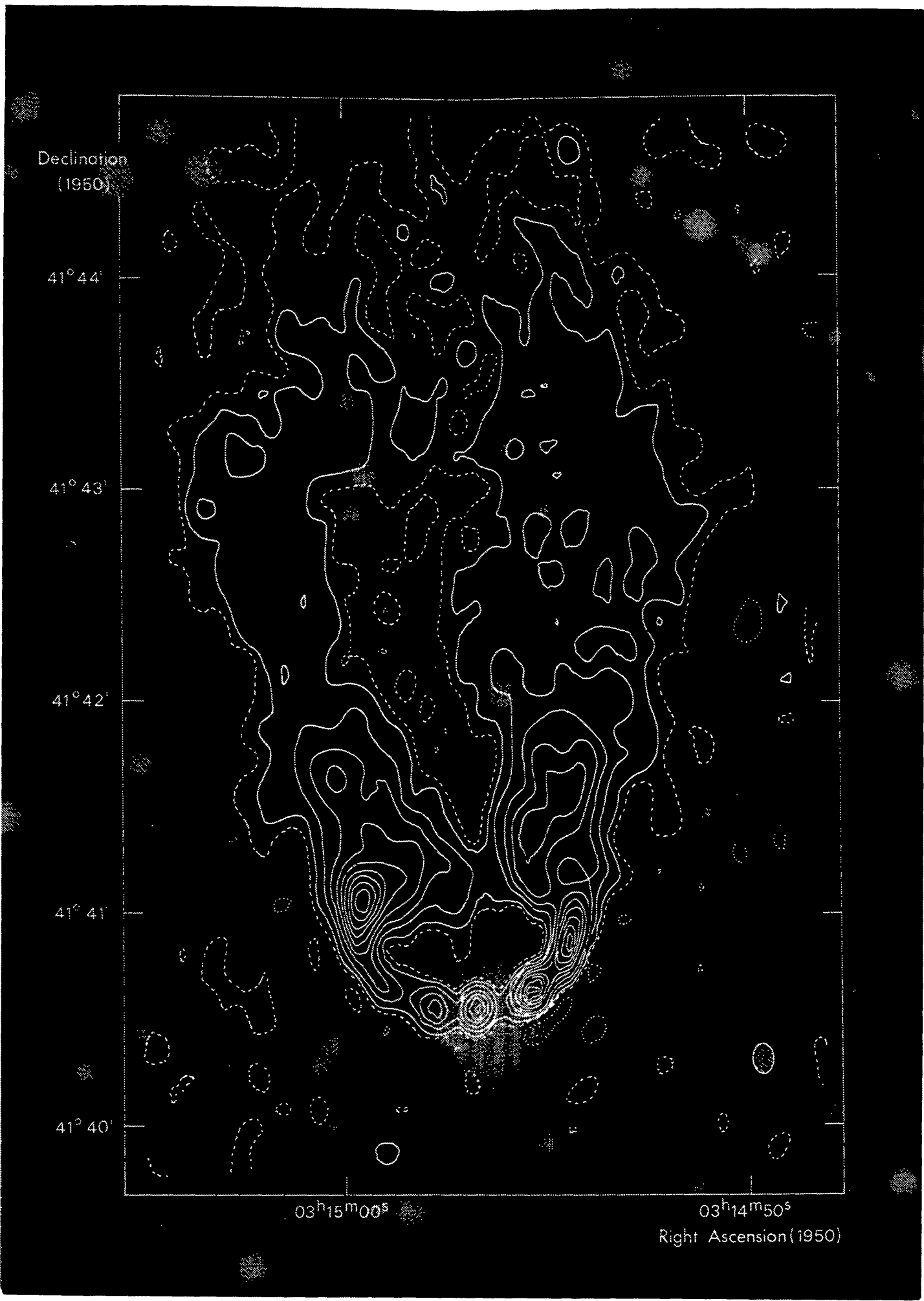

Fig. 6. $5 \mathrm{GHz}$ contour map of the head of NGC 1265 superimposed on the blue Palomar Sky Survey print (from Wellington et al., 1973). 

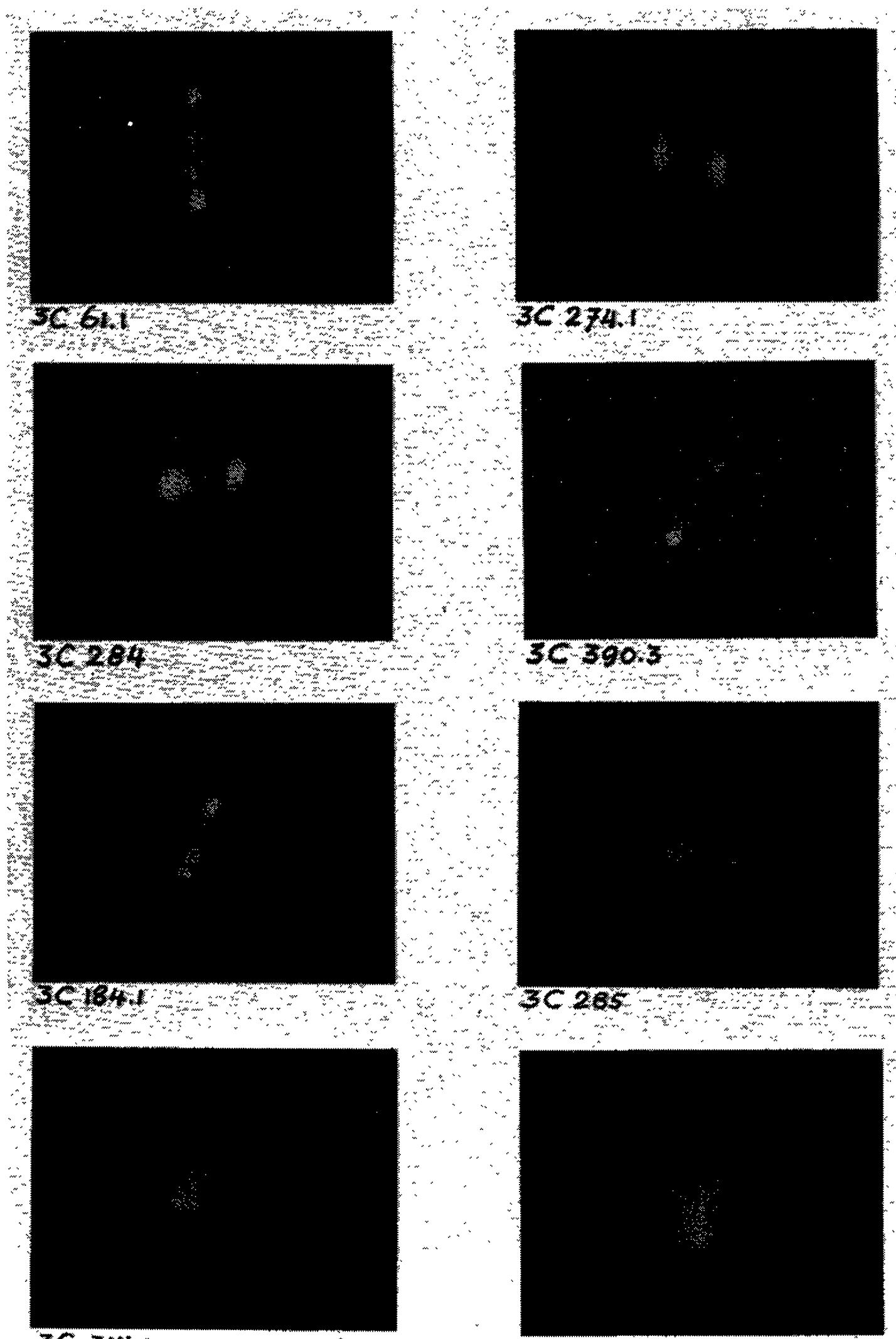

$3 C 314.7$

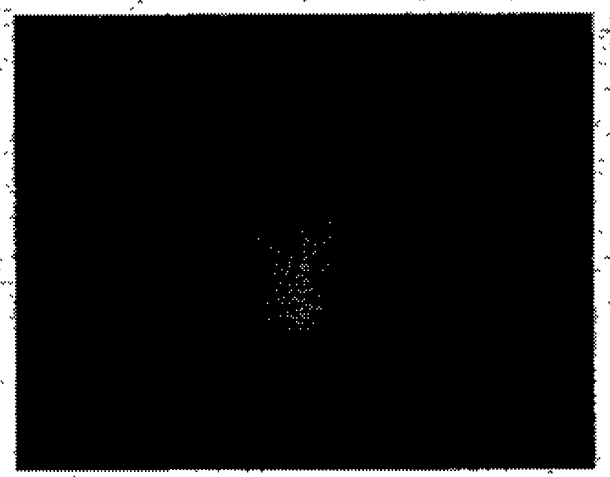

$3<310$

(C) International Astronomical Union - Provided by the NASA Astrophysics Data System 


\section{Conclusions}

From the observations described above it is possible to draw a few tentative conclusions about the nature of head-tail galaxies:

(1) The $5 \mathrm{GHz}$ map of NGC 1265 tells us that the nucleus of this galaxy explodes recurrently every few million years ejecting pairs of radio-emitting plasmoids. Energy releases $\gtrsim 10^{55}$ erg and ejection speeds of a few thousand kilometres per second are involved in each explosion. These ejection speeds are comparable with those now believed to occur in 'normal' radio galaxies (Mackay, 1973).

(2) The polarization and spectral observations suggest that the plasmoids evolve in a basically similar way to that of normal double radio sources, though possibly modified by the magnetosphere of the parent galaxy (Jaffe and Perola, 1973).

(3) The compact components which have been detected in NGC 1265 (Wellington et al., 1973), 3C 129(Riley, 1973) and IC 310 indicate that between major eruptions the nuclei of the galaxies are actively 'simmering'.

The existence of radio trails in clusters provides convincing evidence for the presence of an intra-cluster medium. By assuming that the ram pressure exceeds the internal pressure within the source we can derive a lower limit for the density of the medium. The observations imply densities of at least $10^{-28} \mathrm{~g} \mathrm{~cm}^{-3}$ (Miley et al., 1972; Jaffe and Perola, 1973).

Detailed $5 \mathrm{GHz}$ polarization maps of several head-tail sources are now being reduced. $5 \mathrm{GHz}$ is a sufficiently high frequency that Faraday rotation should be small and the position angles close to those of the intrinsic electric vectors. Preliminary results on NGC 1265 show that the magnetic field in the tail is aligned along the direction of motion. This suggests that the magnetosphere drags behind the galaxy as suggested by Jaffe and Perola. Spectral comparisons should provide further insight into the physical processes involved. We have seen that such observations can yield valuable information on the evolution of radio sources and on the conditions within clusters.

\section{References}

Hill, J. M. and Longair, M. S.: 1971, Monthly Notices Roy. Astron. Soc. 154, 125.

Jaffe, W. J. and Perola, G. C.: 1973, Astron. Astrophys. 26, 423.

Mackay, C. D.: 1973, Monthly Notices Roy. Astron. Soc. 162, 1.

Miley, G. K., Perola, G. C., van der Kruit, P. C., and van der Laan, H.: 1972, Nature 237, 269.

Miley, G. K.: 1973, Astron. Astrophys. 26, 413.

Miley, G. K. and van der Laan, H.: 1973, Astron. Astrophys. 28, 359.

Riley, J.: 1973, Monthly Notices Roy. Astron. Soc. 161, 167.

Fig. 7. $1.4 \mathrm{GHz}$ radio photographs of eight simple double radio galaxies arranged roughly in order of the importance of the high brightness outer edges (from Miley and van der Laan, 1973). From top to bottom the pairs are 3C 61.1 and 3C 274.1, 3C 284 and 3C 390.3, 3C 184.1 and 3C 285, $3 \mathrm{C} 314.1$ and $3 \mathrm{C} 310$.

The brightest spot on each photograph is normalized to the maximum intensity on the map. 
Ryle, M. and Windram, M. D.: 1968, Monthly Notices Roy. Astron. Soc. 138, 1. Wellington, K., Miley, G. K., and van der Laan, H.: 1973, Nature 244, 502.

Willson, M. A. G.: 1971, Monthly Notices Roy. Astron. Soc. 151, 1.

\section{DISCUSSION}

Longair: Some high-resolution (6" arc) observations of sources with radio trails were published several months ago by Julia Riley (Monthly Notices Roy. Astron. Soc. 161, 167, 1973). These indicate that $3 \mathrm{C} 129.1$ is not a radio trail but a rather compact quadruple source centred on the galaxy. In $3 \mathrm{C} 129$ there is a compact source associated with the galaxy, and complex structure in the high brightness regions at the beginning of the tail. It seems therefore that the properties of the trails are not as uniform as implied by Miley. For example, the radio spectral index of the trail in NGC 1265 does not steepen as it does in 3C 129. A catalogue of the differences is given in Riley's paper.

The existence of these compact sources indicates that the galaxies associated with the radio trails are active and the source of energy for the trails. One of the main reasons why we originally proposed that there might be an interaction in these systems is that every time we observe a radio trail, we observe an active radio galaxy of comparable radio luminosity in the same cluster. We do not find examples of isolated radio trails. This feature has still to be explained.

Miley: One is, of course, more likely to find an active galaxy in a cluster than in the general field. One of the problems is that one can only look in sufficient detail at comparatively nearby clusters and the statistics are poor.

Regarding 3C 129.1. I believe that this is indeed a head-tail source seen in projection. Riley's data merely imply that the head is complex, as is also the case for NGC 1265.

Longair: Since we are thinking mainly of radio sources of comparatively low luminosity, there are about 30 cluster sources in the $3 \mathrm{C}$ and $4 \mathrm{C}$ catalogues. In this connection can I ask Wal Sargent if there are redshifts for 3 C 129 or 129.1 yet?

Sargent: No, I have not been able to determine the redshifts of 3C 129 and 3C 129.1 although I have observed both objects. Neither galaxy seems to have emission lines.

E. M. Burbidge: I assume that the density of the intra-cluster gas is too high for it to be cold gas; if it is hot gas, is the density consistent with X-ray measurements?

Miley: The value we derive is consistent with the X-ray measurements and implies temperatures of about $10^{7} \mathrm{~K}$.

Longair: The UHURU observations show that X-ray emission from the Perseus cluster is concentrated around NGC 1275 and does not extend as far as NGC 1265. There is also no definite evidence that the spectrum of the emission is that of thermal bremsstrahlung. It is therefore dangerous to use this X-ray emission as evidence for intergalactic gas in the vicinity of NGC 1265 . 